\title{
Taller de Activación Urbana TAU
}

\author{
Guillermo Moya Rodríguez \\ Jimmy Morales López
}

Reseña de Taller de Activación Urbana

Invitados nacionales

Institución: Universidad de Costa Rica

E-mail: Guillermo Moya Rodríguez: guillemoya21@ gmail.com

Jimmy Morales López: jimgerjim@gmail.com

Recibido: 10 de noviembre de 2018

Aprobado: 11 de diciembre de 2018

\section{Guillermo Moya Rodríguez}

Estudiante de Arquitectura, Universidad de Costa Rica. Estudiante de Ingeniería Civil, Universidad Fidelitas. Fundador de Morok Design Studio.

\section{Jimmy Morales López}

Estudiante de Arquitectura, Universidad de Costa Rica Rica. Actualmente labora como asistente de investigación en la misma unidad. Ha colaborado como asistente de investigación en temas de vejez y patrimonio arquitectónico.

\section{Resumen:}

En agosto del 2018 se llevó a cabo la séptima edición del Taller de Activación Urbana (TAU). A saber, el Taller plantea un espacio de experimentación, arte y discusión sobre la forma de habitar nuestros espacios públicos, desde una perspectiva de activación urbana por parte de la ciudadanía. Este año se presentó como tema específico "Ciudad y Género, desde un enfoque feminista"; se trabajó sobre la línea temática de "Integración de Bordes Urbanos" específicamente en el límite sur de la Ciudad Universitaria Rodrigo Facio. El Taller contó con la participación de seis facilitadoras (es) internacionales y siete nacionales que favorecieron la concreción de distintas propuestas enfocadas a la activación del espacio mediante la reflexión y construcción colectiva de procesos participativos para la autogestión del espacio público en todas sus dimensiones.

Palabras clave: espacio público; participación; acción participativa, ciudad y género.

\section{Urban Activation Workshop 2018}

\begin{abstract}
:
In August 2018 the seventh edition of the Urban Activation Workshop (TAU, by its initials in Spanish) was held. Namely, the Workshop proposes a space for experimentation, art and discussion on how to inhabit our public spaces, from a perspective of urban activation by citizens. This year, the theme "City and Gender, from a Feminist Perspective" was presented as a specific theme; we worked on the thematic line of "Integration of Urban Borders" specifically in the southern limit of the Ciudad Universitaria (University City) Rodrigo Facio. The workshop counted with the participation of six international facilitators and seven nationals that favored the realization of different proposals focused on the activation of space through reflection and collective construction of participatory processes for self-management of public space in all its dimensions.
\end{abstract}

Keywords: public space; participation; participative action; city and gender. 
D

esde el año 2009 la fundación Pausa Urbana ha generado y promovido una serie de estrategias y actividades enfocadas a la construcción de espacios que fortalezcan los vínculos entre las personas y sus espacios públicos desde un enfoque de hábitat, integrando el plano físico con el simbólico. Desde este marco conceptual nace el Taller de Activación Urbana (TAU), entendiendo el concepto de activación urbana como "el proceso de construir, dar significado y consolidar el vínculo vital y cotidiano entre la gente y sus espacios públicos" (Villalta, 2015), aplicándolo como estrategia metodológica de procesos de gestión participativa de espacios públicos. Estos son entendidos como desencadenantes de condiciones que propicien la permanencia, el uso y la apropiación por parte del colectivo que los habita. El Taller es dirigido por Pausa Urbana, realizado en conjunto con la Escuela de Arquitectura de la Universidad de Costa Rica e inscrito en la Vicerrectoría de Acción Social, de la cual recibe apoyo económico para su gestión.

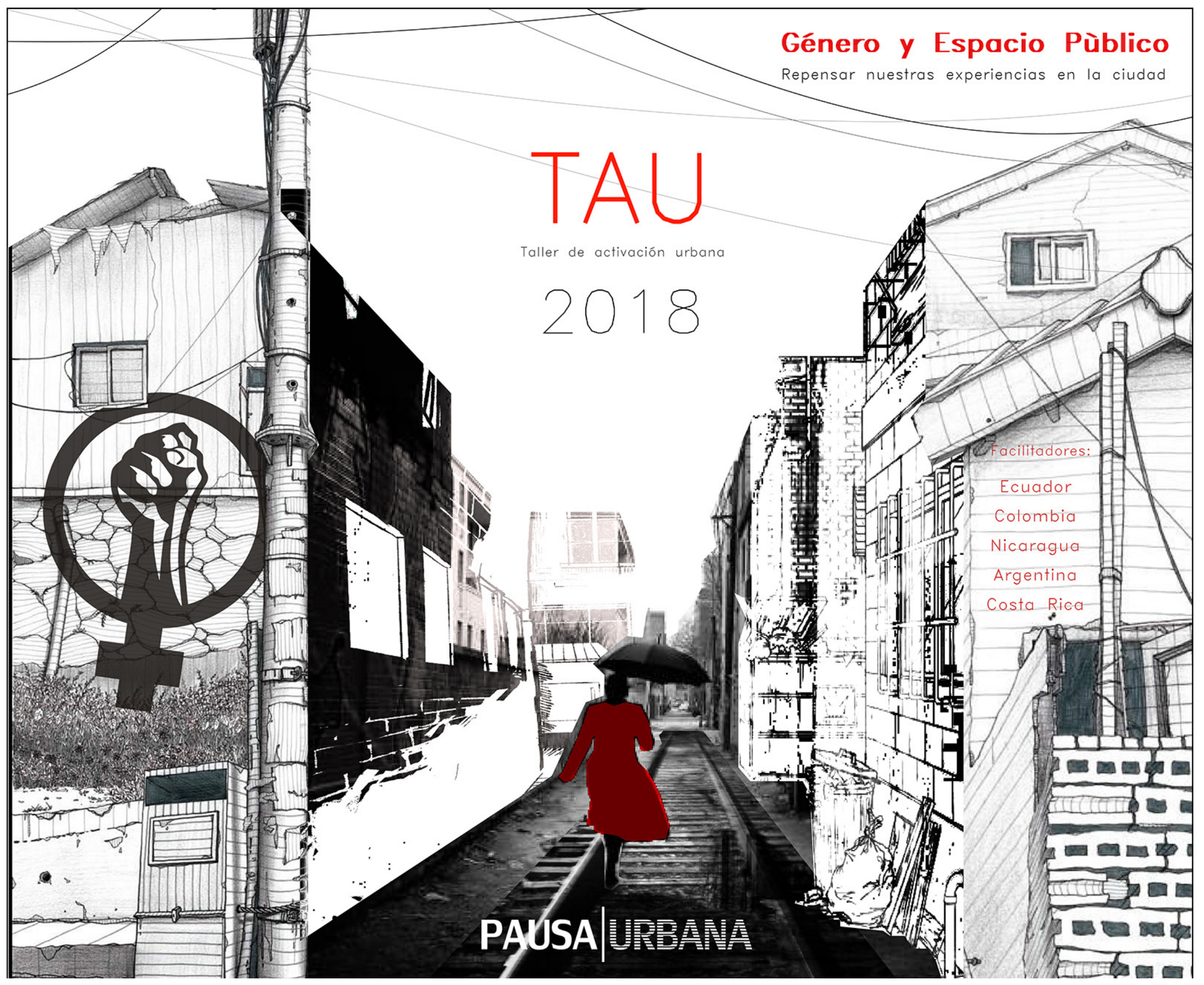

Figura 1. Afiche del TAU 2018 realizado por Mariana Urrutia.
A La séptima edición del Taller de Activación Urbana se llevó a cabo entre el 20 y el 25 de agosto del 2018, en donde se presentó como tema específico "Ciudad y Género, desde un enfoque feminista", trabajado sobre una de las tres líneas temáticas del Taller, para este caso la de "Integración de Bordes Urbanos" (las otras dos líneas son "Espacios Públicos Comunitarios" y "Espacios Públicos en Centros Urbanos"). La zona escogida para la realización del Taller fue sobre el límite sur de la Ciudad Universitaria Rodrigo Facio donde la franja definida como retiro de vía para el paso del Tren Urbano se suele entender como un espacio residual que atraviesa la ciudad, pero al que las edificaciones (y también instituciones públicas como la misma UCR) le dan "la espalda", generando una zona ambigua de borde. Si bien este espacio es ampliamente usado por peatones como atajo o zona de paso, este presenta condiciones de inseguridad y poco acondicionamiento físico para tal fin, situación de vulnerabilidad que afecta principalmente a las mujeres que caminan solas. Esto se pudo comprobar ampliamente durante el desarrollo del Taller y sus distintas actividades. 


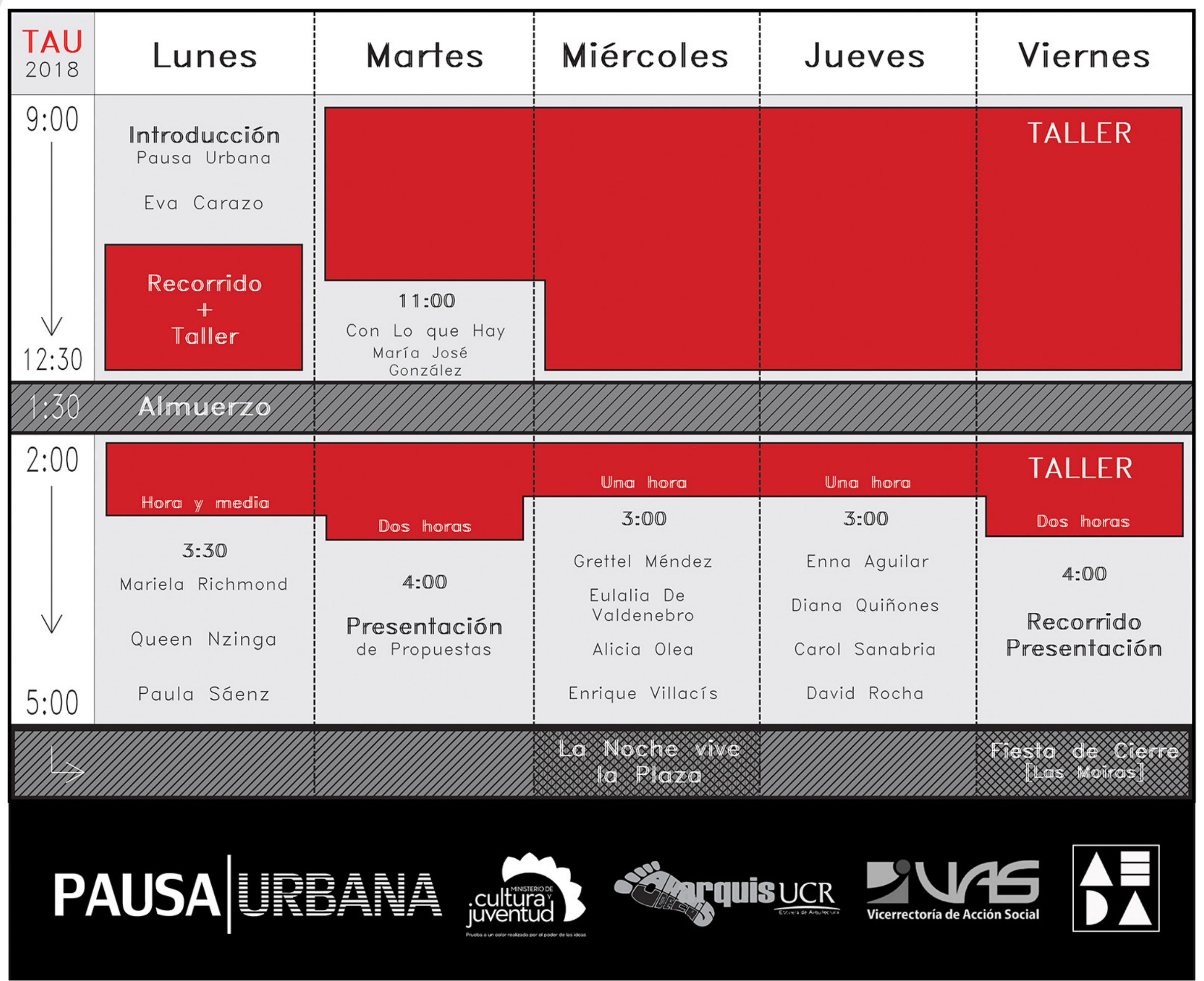

Figura 2. Programa del Taller elaborado por Pausa Urbana.
Para esta edición del Taller, se logró gestionar la participación de seis facilitadoras (es) internacionales y siete nacionales:

Paula Sáenz, politóloga y gestora cultural de procesos sociales (Colombia).

Enrique Villacís, arquitecto y docente, procesos participativos para la construcción de infraestructura comunitaria (Ecuador).

Diana Quiñones, diseñadora objetual y meditadora profesional (Colombia).

Eulalia De Valdenebro, artista, docente e investigadora (Colombia).

David Rocha, antropólogo, escritor, dramaturgo y cuentero (Nicaragua).

Alicia de Olea, colectivo Del Mate, circo y activista feminista (Argentina).

Maria José González, circo y gestora cultural (Costa Rica).

Eva Carazo, socióloga y gestora social (Costa Rica).

Mariella Richmond, artista y gestora cultural (Costa Rica).

Grettel Méndez, artista escénica, dramaturga y activista permorfer (Costa Rica).

Ena Aguilar, bailarina, coreógrafa y panadera (Costa Rica).

Carol Sanabria, arquitecta, tallerista de cuerpo y espacio, actriz y bailarina (Costa Rica).

Queen Nzinga, poeta y gestora cultural (Costa Rica).

Ellos fueron quienes conformaron el equipo de facilitadores, talleristas y conferencistas durante los distintos procesos creativos, discusiones y conversatorios. Se encargaron de guiar los seis módulos de trabajo en equipo y sus procesos metodológicos, que favorecieron la concreción de distintas propuestas enfocadas a la activación del espacio 
trabajado desde un enfoque género y ciudad. Se trabajó frontalmente la percepción y condición del espacio con respecto a temas como el acoso callejero y espacios seguros para todos y todas. También se articularon las distintas propuestas con vecinos como Fundevi, el centro cultural Las Moiras, la parada del Tren Urbano, entre otros.

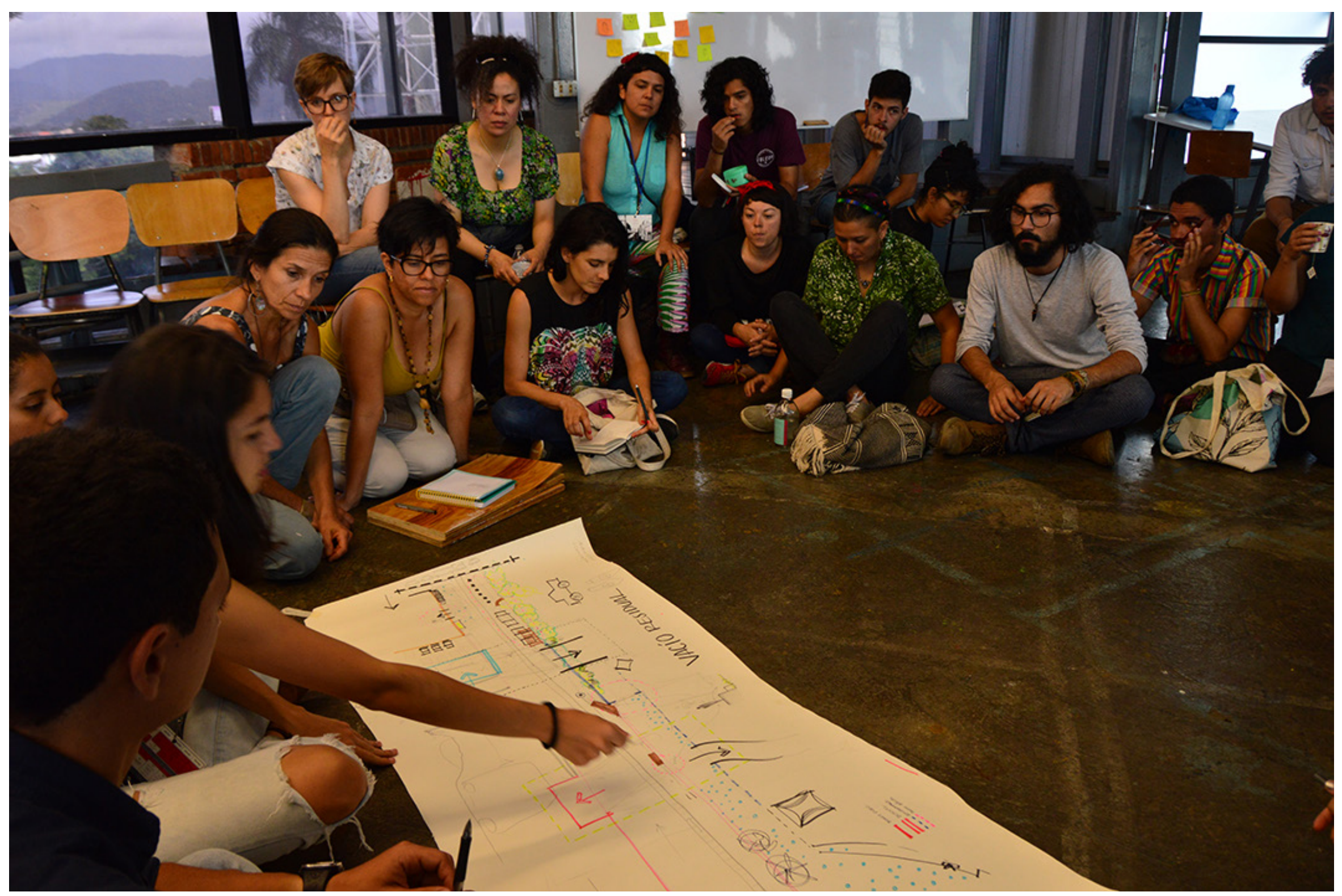

Figura 3. Proceso de taller TAU 2018, fotografía A por Jaire Cagigal Camacho

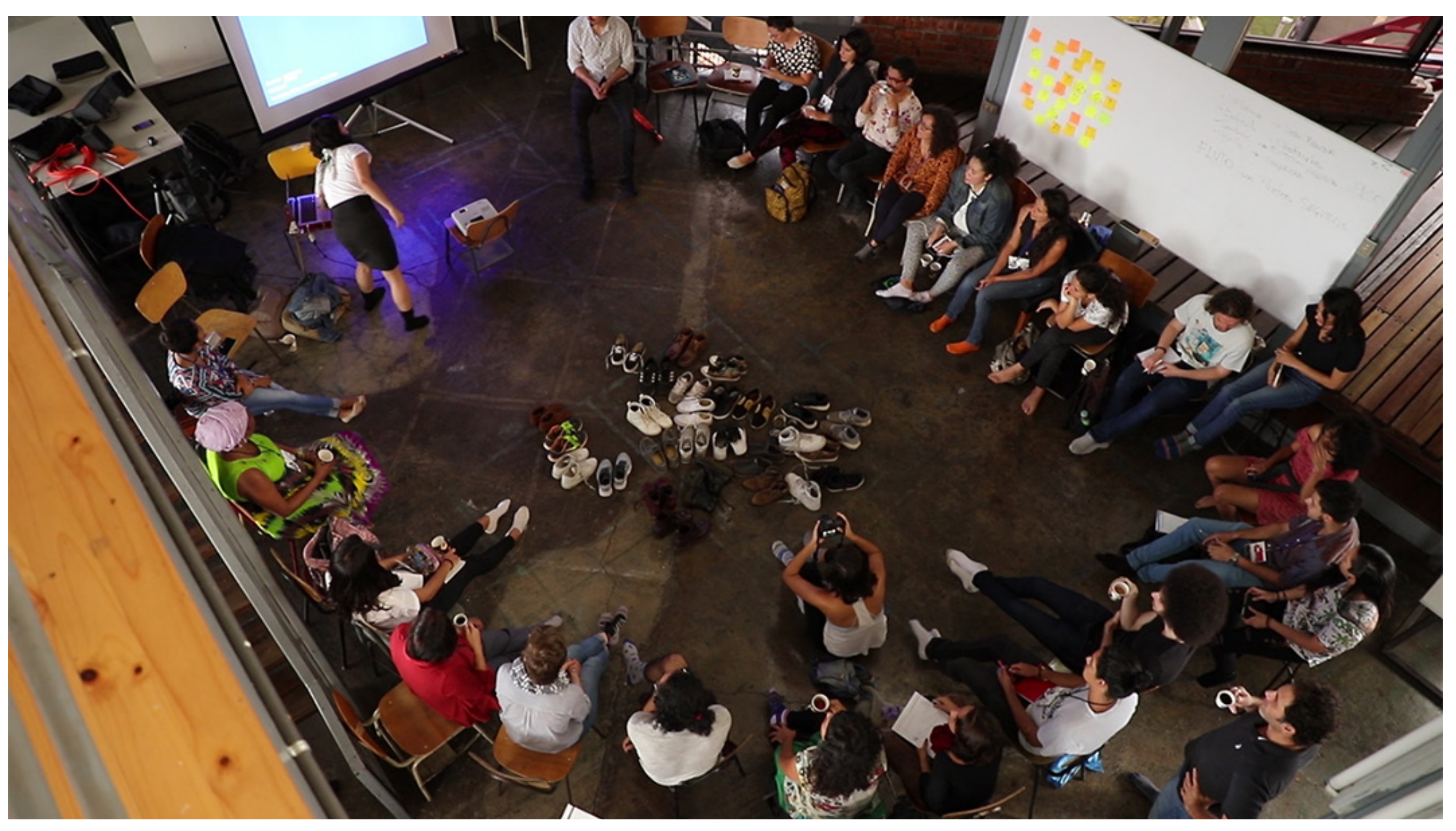

Figura 4. Proceso de taller TAU 2018, fotografía por Manduca Audiovisual. 
Figura 5. Frase de una transeúnte. Manduca Audiovisual.

Figura 6. Elaboración propia basada en fotografía de Manduca Audiovisual.
La dinámica del TAU se enfoca en la reflexión y construcción colectiva de procesos participativos para la autogestión del espacio público en todas sus dimensiones. Esto permite que participantes y facilitadoras (es) establezcan horizontalmente estrategias puntuales mediante las cuales se pueda activar oportunamente el espacio y cuya repercusión contribuya en el corto, mediano y largo plazo a su mejoramiento y apropiación por parte de sus habitantes. En esta edición, se desarrollaron propuestas enfocadas a la señalética, la acción performática, una campaña en redes sociales y a una serie de acciones de mejora física del espacio para el tránsito peatonal. Estas se describen a continuación:

\section{Señalética}

Esta tuvo un impacto muy fuerte a nivel espacial y territorial, puesto que la intención inicial fue evidenciar la unión emergente entre mujeres en lazos de cooperación para demostrar, denunciar y erradicar conductas de acoso sexual en el espacio público. La construcción de la acción se basó en la recolección de información de personas que transitan el espacio comprendido entre el campus universitario y las vías del tren. Se recolectó información para establecer puntos críticos para la realización de intervenciones asociadas y así evidenciar los peligros a los cuales las mujeres se encuentran expuestas al transitar por dicha zona. Se logró una gran participación por parte de la gente que transitaba por la zona.
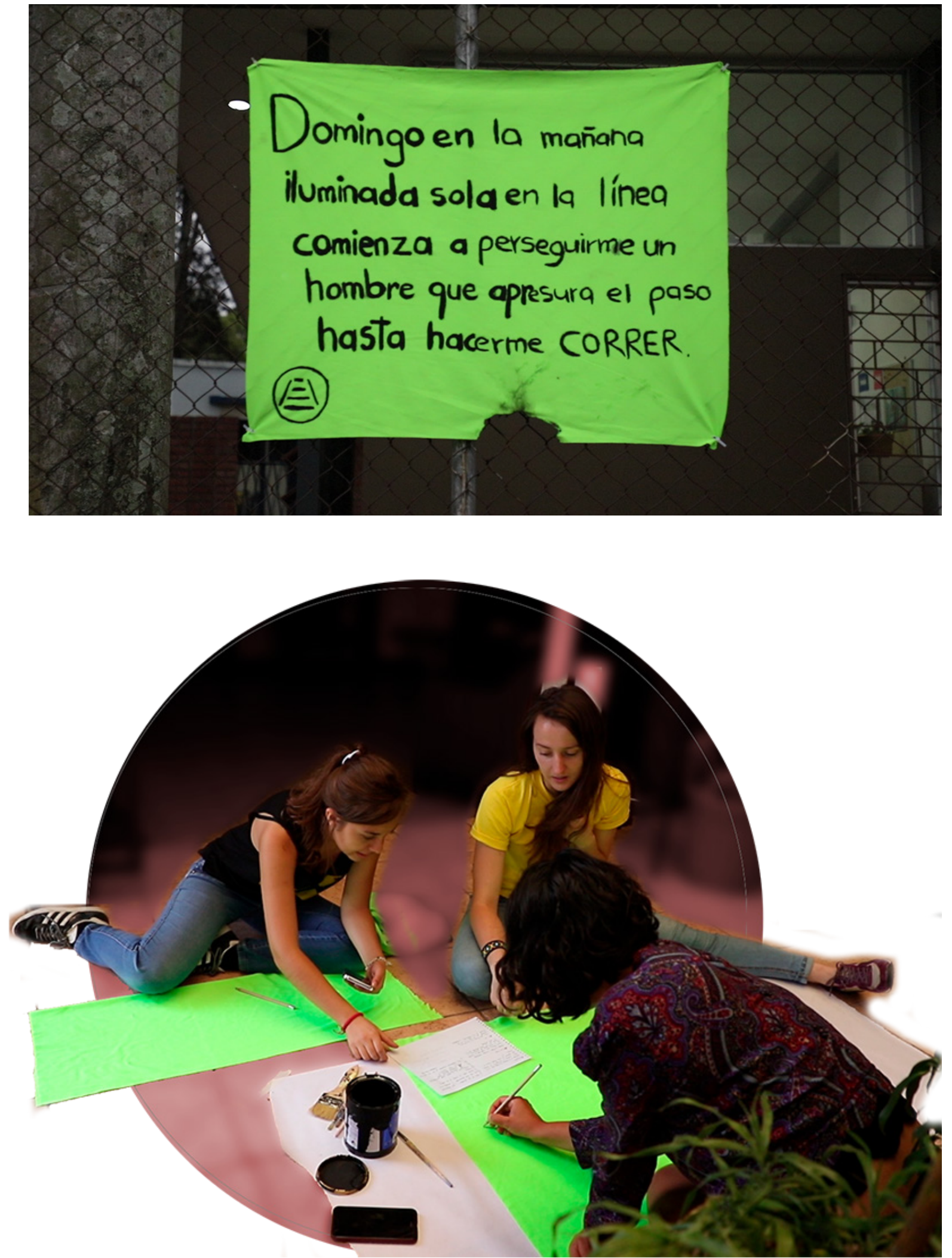


\section{Acción performática}

Las actividades planeadas en el ámbito de la acción performática involucraron la apropiación del espacio a nivel simbólico, siendo el principal énfasis de estas la evidencia de la pérdida del espacio colectivo y la discusión sobre el papel que ocupan las mujeres en los bordes urbanos y el espacio público. La dinámica se basó en la construcción de trajes que exageraban gestualmente los principales roles de género que la sociedad le impone a la mujer. Esto con el fin de reflexionar y evidenciar la idea de la mujer como partícipe del espacio público ante las constantes situaciones a las cuales se ve sometida, dadas las conductas acosadoras normalizadas que rigen en estos espacios.

Figura 7. Elaboración propia basada en fotografía de Jaire Cagigal Camacho.

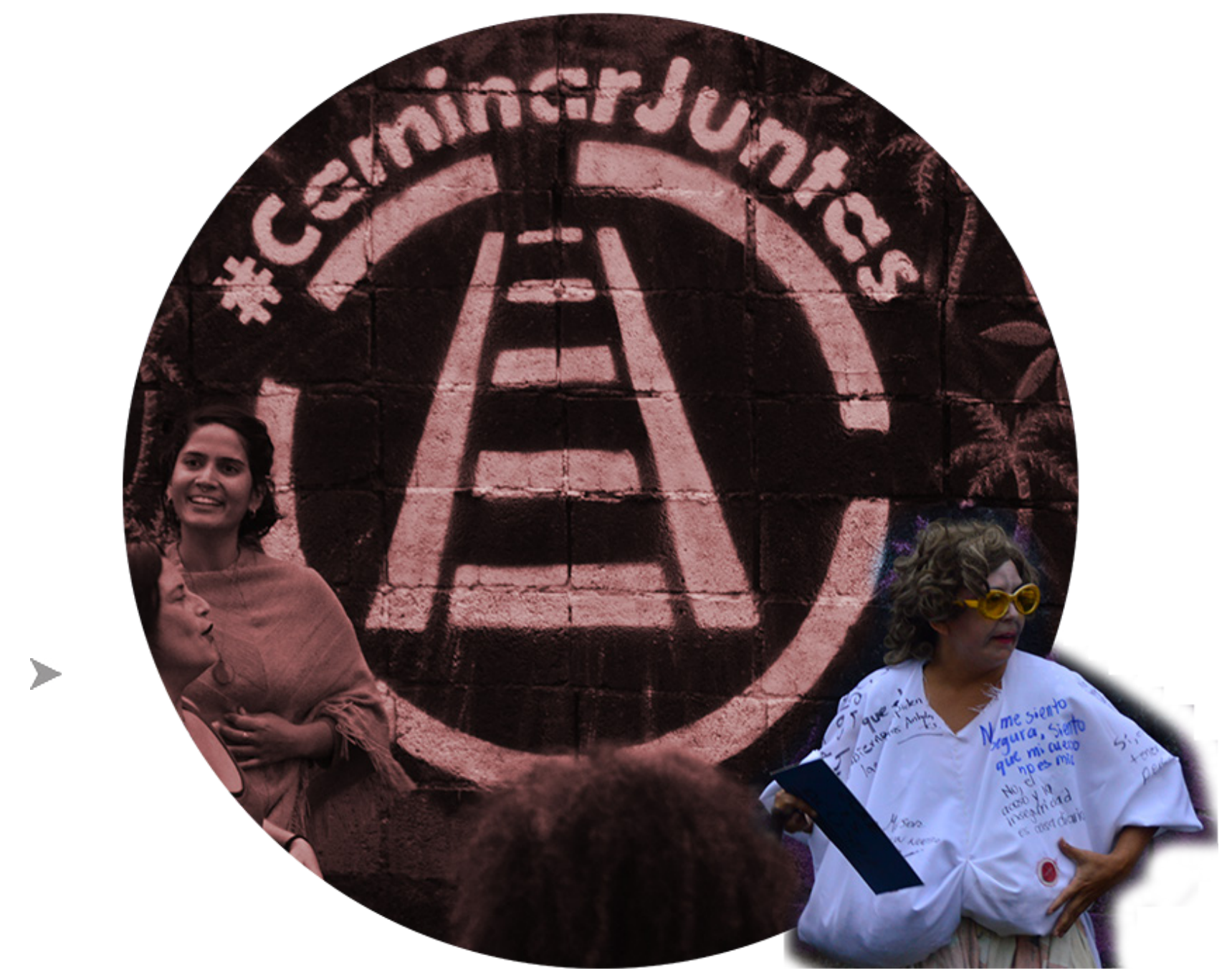

Figura 8. Fotografías de Manduca Audiovisual. $\quad$

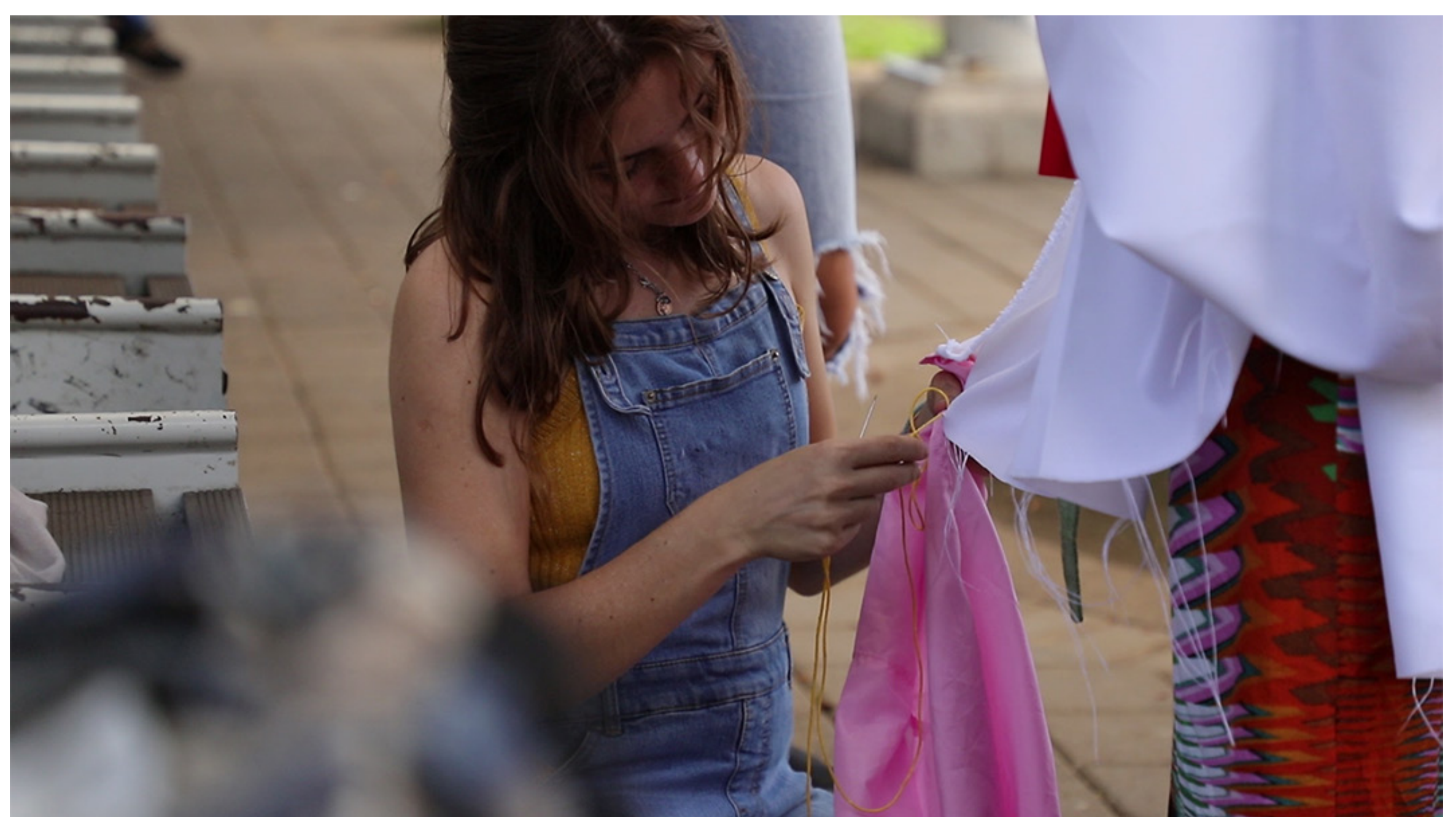




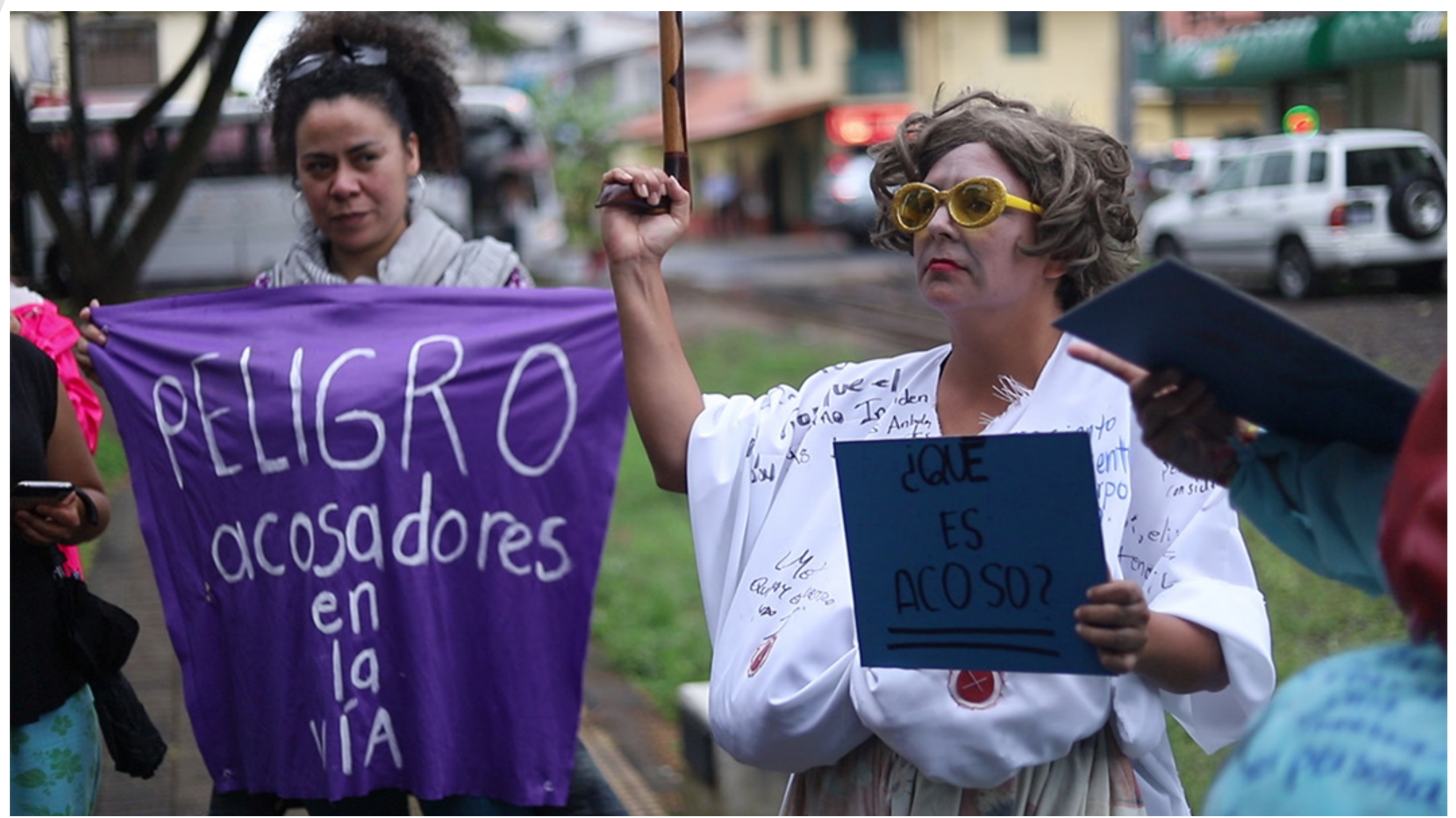

Figura 9. Fotografías de Manduca Audiovisual.

Campaña en redes sociales "Caminar Juntas"

Se decidió trabajar una estrategia de comunicación sobre el tema de acoso callejero, para lo que se diseñó y creó la campaña en redes sociales "Caminar Juntas" con el fin de evidenciar los procesos y acciones realizadas en el espacio durante la semana del Taller, pero con la intención de darle continuidad al movimiento que se logró y, de esta manera, se puedan seguir haciendo y compartiendo acciones similares, articuladas sobre el mismo lema, tanto en este, como en otros espacios. De esta manera, el Taller generó un resultado de activación sustentable que permite ir creciendo con el tiempo, de acuerdo con el marco conceptual explicado anteriormente.

Figura 10. Elaboración propia basada en fotografía de Manduca Audiovisual.

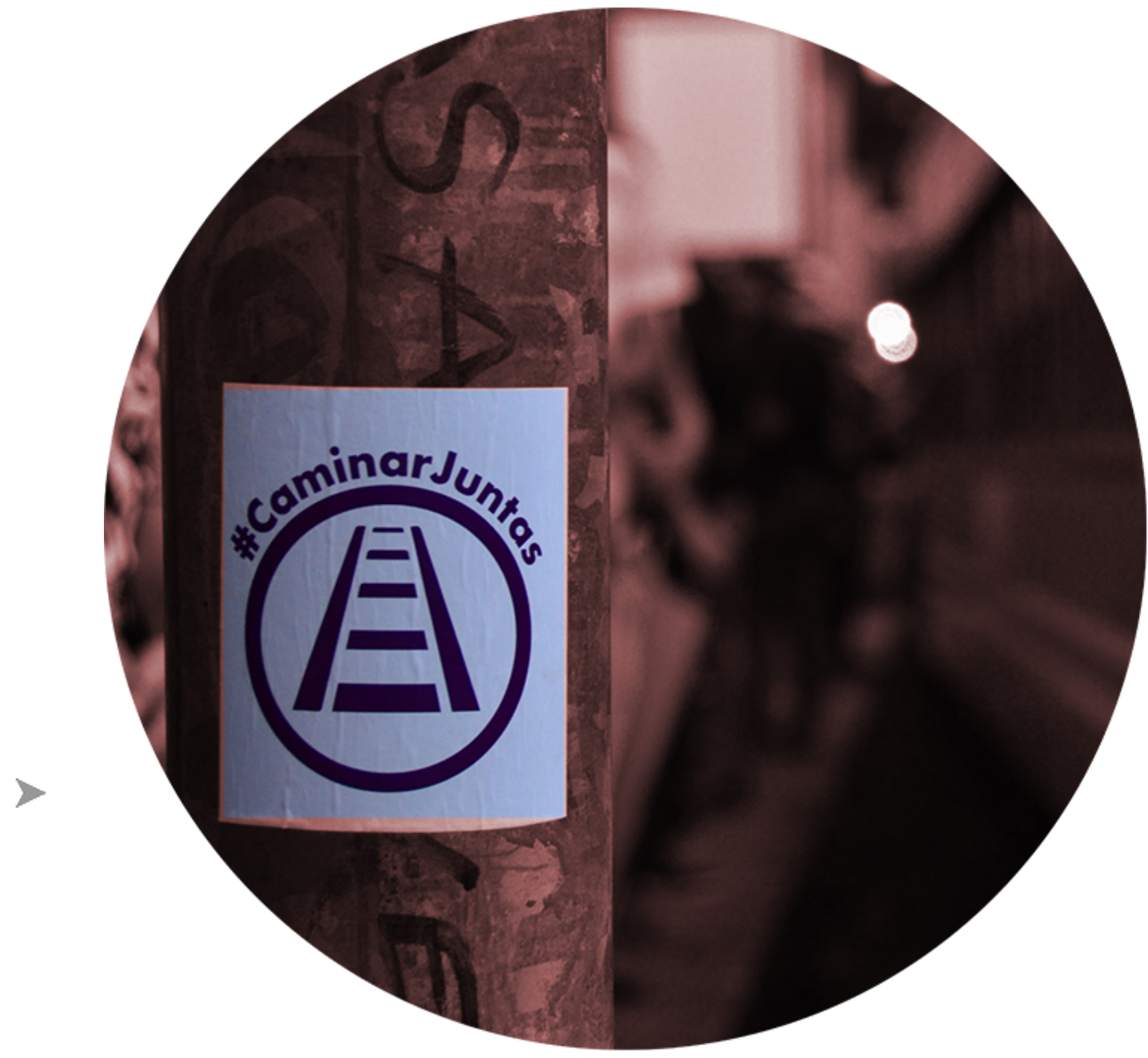




\section{Acondicionamiento físico de la red peatonal y creación de un espacio de estar}

Durante el Taller, se identificó que, a pesar del fuerte uso del espacio como ruta peatonal alternativa, este no reúne las cualidades apropiadas para permitir un transito seguro, cómodo e inclusivo. Por lo tanto se decidió, en la medida de lo posible, mejorar algunas de sus condiciones. Se desarrolló una rampa de lastre y de materiales pétreos para articular el paso hacia la acera de la parada del tren, se protegió un árbol aledaño mediante un espacio tipo jardinera y se eliminaron algunos obstáculos. También se potenció un espacio planteado por Fundevi hace algunos meses. La gestión de materiales y herramientas para esto fue resuelta por los mismos participantes del Taller, utilizando lo encontrado en la zona y acudiendo a los vecinos del lugar, como la misma Fundación UCR.

Figura 11. Elaboración propia basada en fotografía de Manduca Audiovisual.

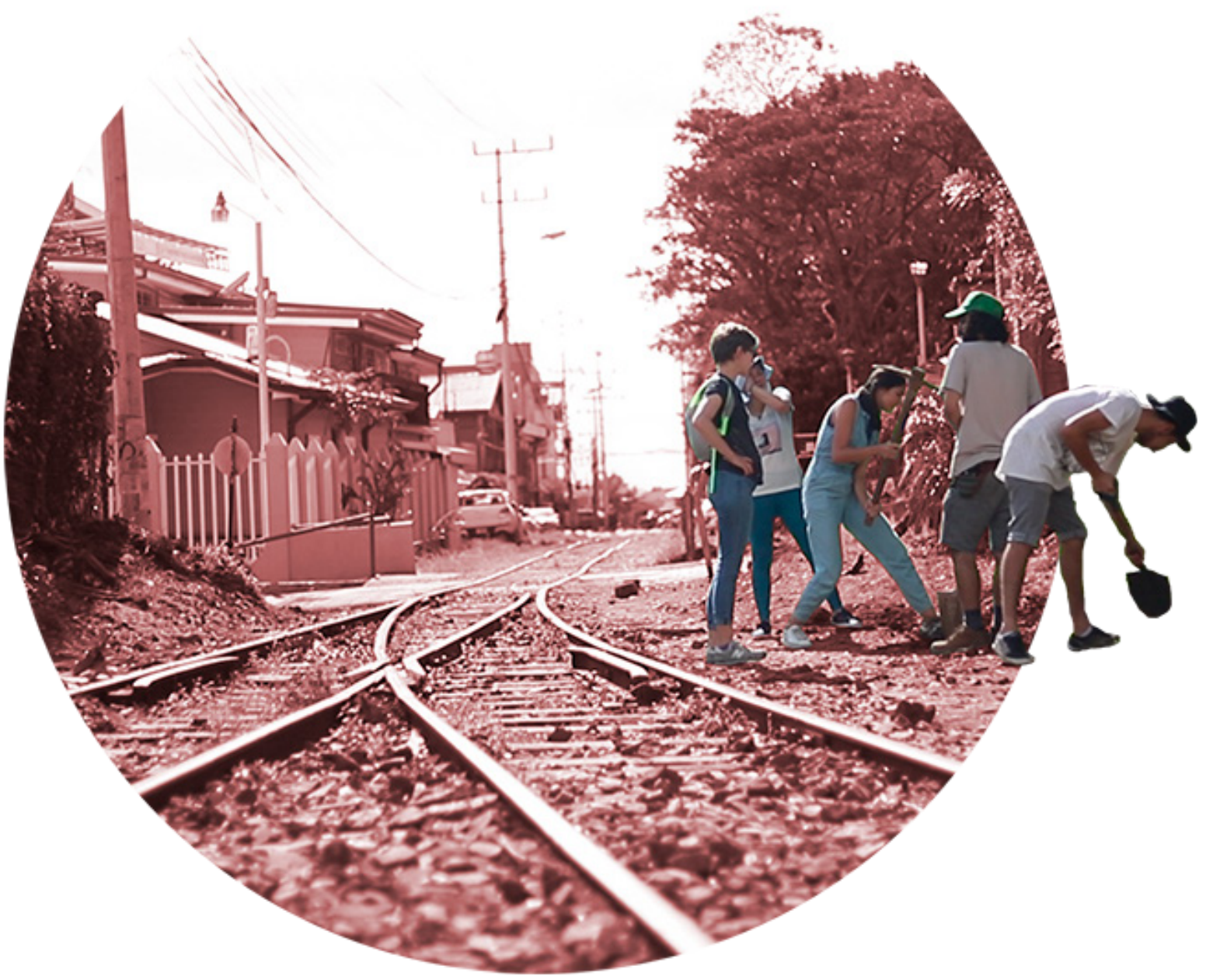

Figura 12. Fotografía de Manduca Audiovisual.

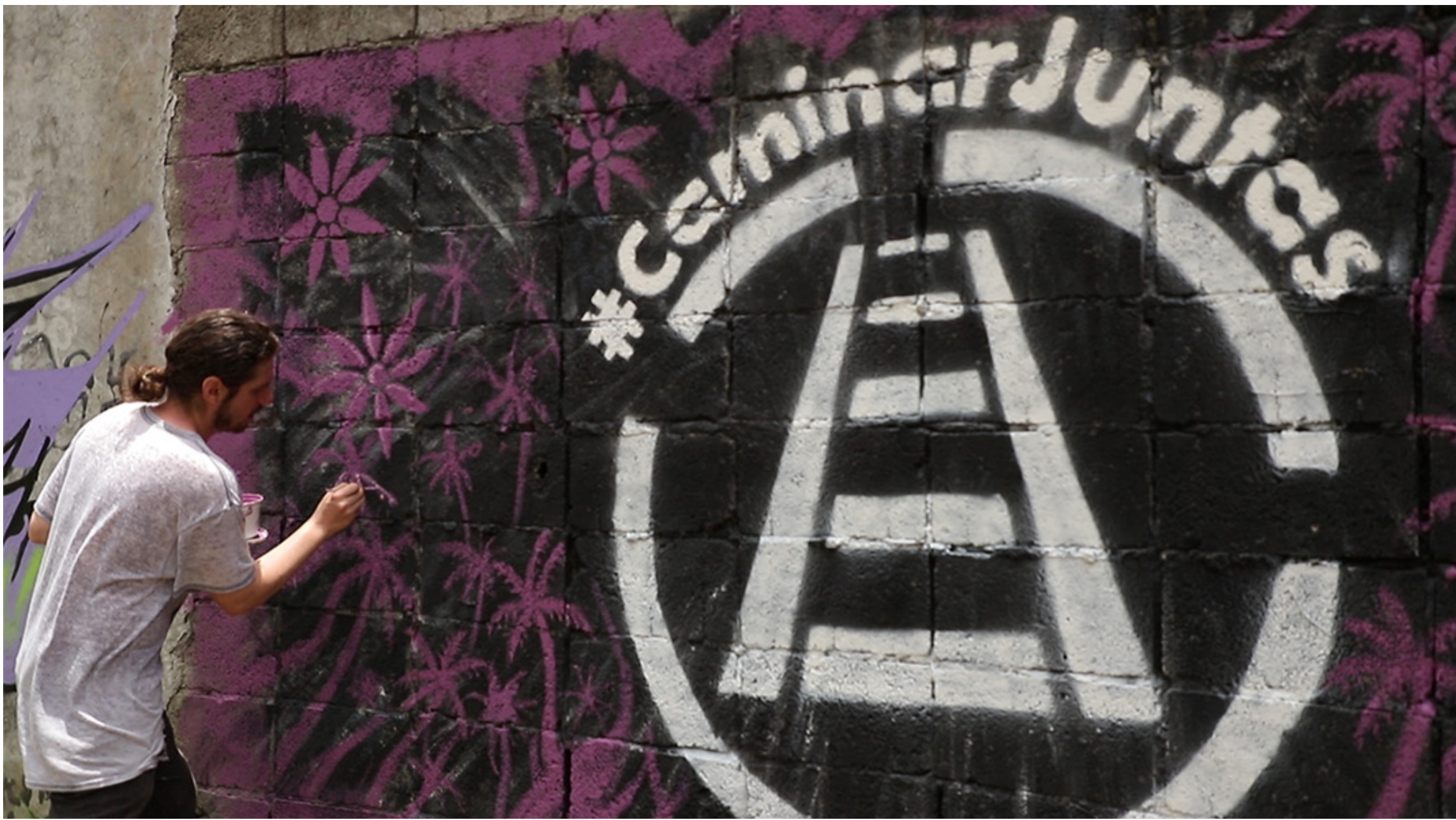


Figura 13. Fotografía de Jaire Cagigal Camacho.

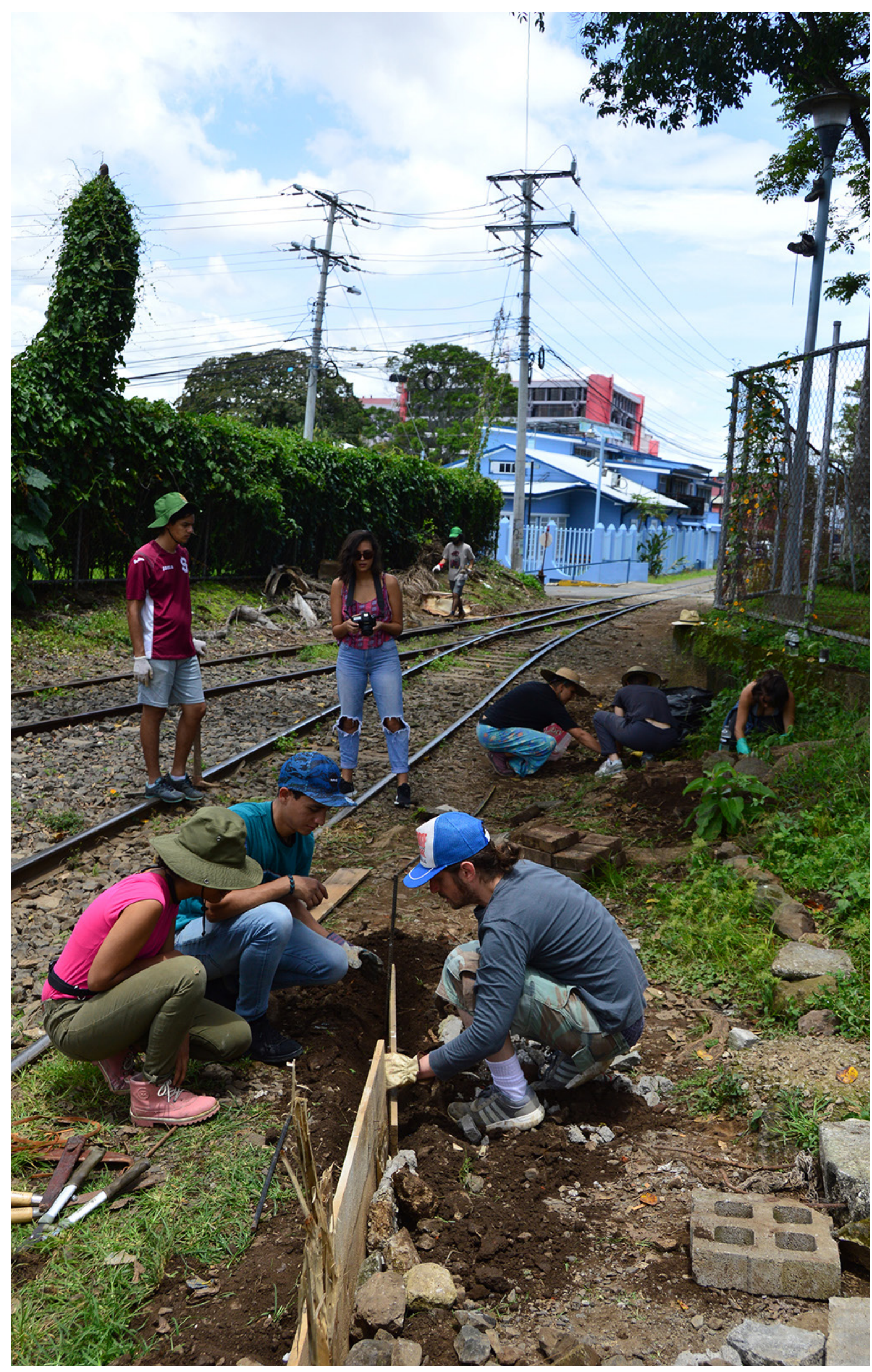

Una situación que emergió durante el desarrollo del Taller fue la identificación de un punto de acoso ubicado en el extremo oeste del tramo donde se trabajó, frente a la cocina del restaurante "La Parrillita de Pepe", de la cual salian varios hombres a acosar a las mujeres que pasaban diariamente. Esto se pudo corroborar por varias de las mujeres que llevaban el Taller y que habían sido acosadas en ese punto. Ante esto, se realizó primero la acción de señalar el punto de acoso mediante un grafiti en el suelo frente al lugar. Este gesto fue inmediatamente borrado por los trabajadores del restaurante, pero permitió la oportunidad de enfrentarlos directamente de forma pacífica para hacerles ver la incomodidad de las y los transeúntes. Luego, al cierre del Taller, todo el grupo de participantes junto con las personas invitadas para esta actividad realizaron una acción conjunta frente a este punto, cantando consignas reivindicativas del feminismo y compartiendo en voz alta pensamientos acerca del tema de acoso callejero. En este sentido, el Taller generó diálogo y contacto con muchas personas que lo habitan y recorren diariamente, exponiendo la condición, la gravedad y el impacto que tiene el acoso en las mujeres que lo padecen y, por ende, en la sociedad y en el espacio público. 


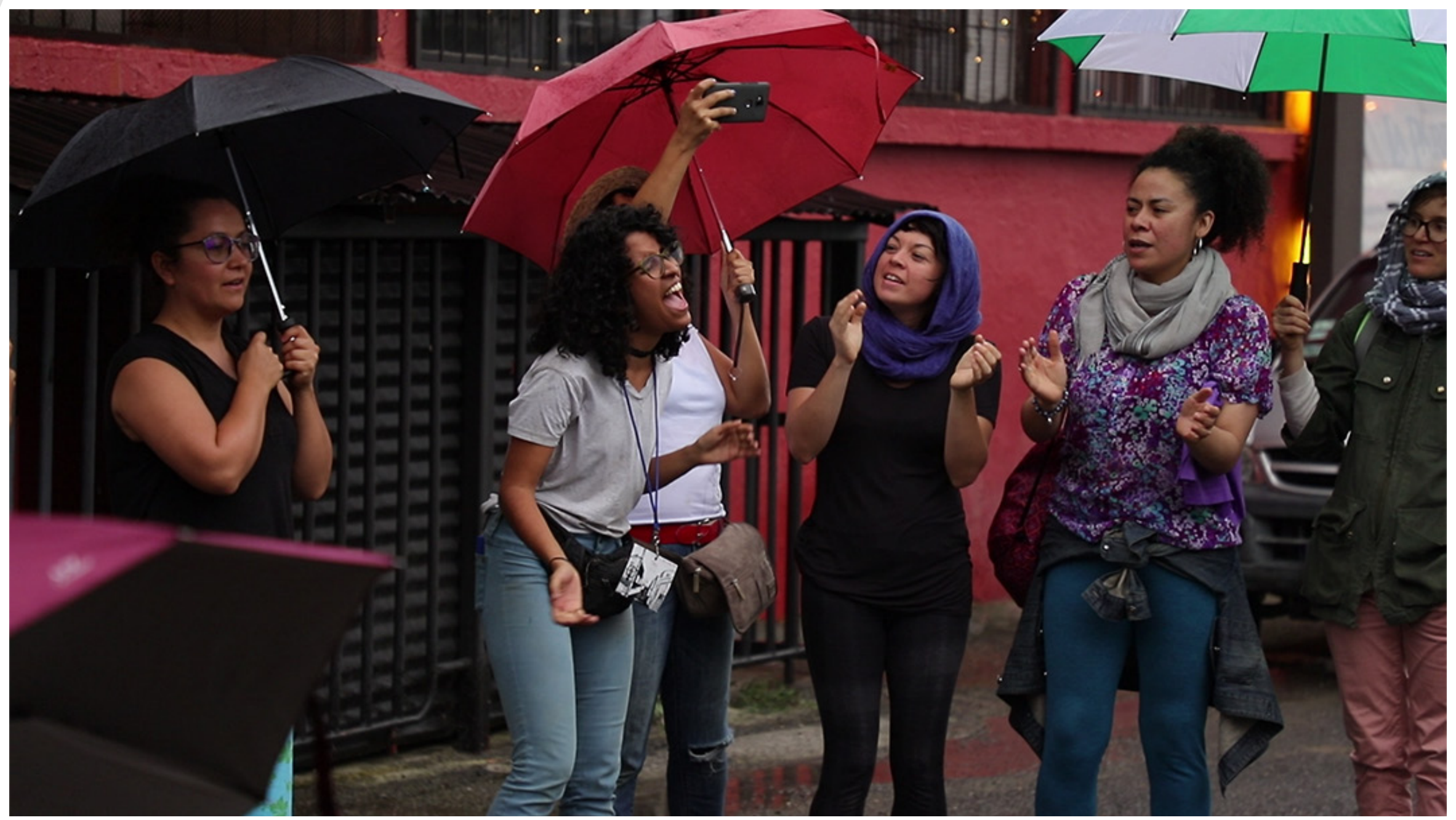

Figura 14. Actividad de cierre, fotografía por Manduca Audiovisual.
Además de todas estas acciones desarrolladas durante el Taller se pudo construir fraternalmente una red de trabajo entre todas las personas participantes, quienes siguen en contacto y apoyando colectivamente otras acciones e iniciativas. Cabe resaltar que además de las facilitadoras(es) extranjeros, llevaron el Taller cuatro estudiantes avanzados de la Universidad Católica de Ecuador, quienes vinieron a presentar el trabajo del Taller Con Lo Que Hay, dirigido por el arquitecto Enrique Villacís. De este modo, se dio una importante interacción y convivencia durante la semana con resultados, reflexiones personales y aprendizajes muy importantes para todas las personas participantes. Por último, el próximo TAU está proyectado para agosto del 2019 y tendrá como eje temático los espacios colectivos comunitarios, para lo que se trabajará en algún barrio o comunidad urbana. 